
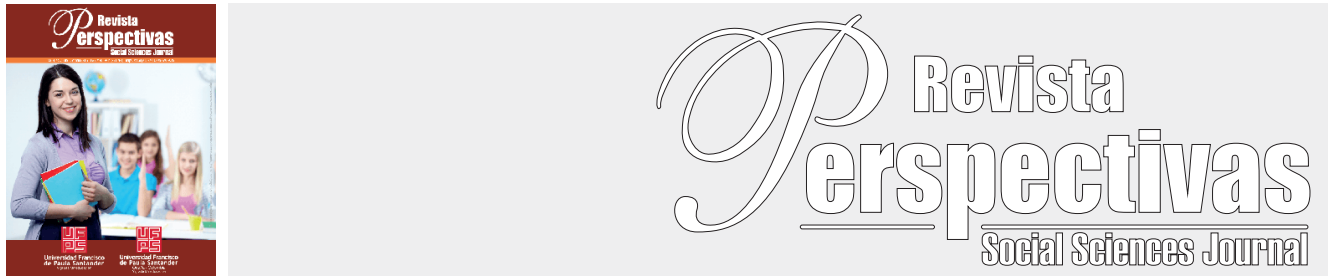

Artículo Original

https://doi.org/10.22463/0122820X.1793

\title{
EI dilema de la guerra o la paz: representaciones sociales sobre la paz de jóvenes del colegio San José de Cúcuta
}

The dilemma of war or peace: social representations on peace by young people from San José School in Cúcuta

\begin{abstract}
Maritza Carolina Jaimes-Márquez ${ }^{1}$, Laura Nataly Galvis-Velandia², Miguel Augusto Güiza-Castellanos ${ }^{3}$
'Magister en Paz, Desarrollo y Resolución de Conflictos, maritzacarolinajm@ufps.edu.co, ORCID 0000-0002-4728-6064, Universidad Francisco de Paula Santander, Cúcuta, Colombia.

${ }^{2}$ Magister en Educación, lauranatalygv@ufps.edu.co, ORCID 0000-0003-2444-8398, Universidad Francisco de Paula Santander, Cúcuta, Colombia.

${ }^{3}$ Magister en Práctica Pedagógica, miguelaugustogc@ufps.edu.co ORCID 0000-0003-1918-456X, Universidad Francisco de Paula Santander, Cúcuta, Colombia.
\end{abstract}

Cómo citar: M. Jaimes y L. Jaimes, M. Güiza "El dilema de la guerra o la paz: representaciones sociales sobre la paz de jóvenes del colegio San José de Cúcuta”. Perspectivas, vol. 4, no. 2, pp. 31-38, 2019.

Recibido: Junio 27, 2018; Aprobado: Octubre 03, 2018.

\begin{tabular}{ll}
\hline RESUMEN \\
\hline Pabras clave: & El presente artículo expone el desarrollo y resultados de la investigación que tiene como título el dilema de la \\
guerra o la paz: representaciones sociales sobre la paz de jóvenes del colegio san José de Cúcuta, el objetivo \\
general fue Comprender las Representaciones Sociales de paz de los jóvenes estudiantes de la institución \\
Educación & educativa San José de Cúcuta como fundamento a una práctica pedagógica. Las teorías aplicadas fueron las \\
Guerra & representaciones sociales, los modelos pedagógicos y una mirada hermenéutica en la práctica pedagógica, que \\
& desde la rigurosidad permiten dar una mirada crítica frente a la investigación, asimismo, se fundamenta en \\
& una investigación cualitativa, con un diseño hermenéutico, encaminada a dar respuesta a los interrogantes aquí \\
& generados y los objetivos mencionados con anterioridad.
\end{tabular}

\section{ABSTRACT}

Keywords:

The present article presents the development and results of the research that has as its title the dilemma of war or peace: social representations of peace by young people from the San José de Cúcuta school. The general

Peace

Pedagogy

Education

War objective was to understand the social representations of peace by young students from the San José de Cúcuta educational institution as a basis for a pedagogical practice. The theories applied were social representations, pedagogical models and a hermeneutic look at pedagogical practice, which from a rigorous point of view allow for a critical look at the research. Likewise, it is based on a qualitative research, with a hermeneutic design, aimed at answering the questions generated here and the objectives mentioned above.

\section{Introducción}

Abordar el conflicto en el contexto colombiano tiene asociaciones directas con manifestaciones de la violencia que por décadas ha afectado a millones de personas en el país. Por ende, el tema central de la investigación se centró en la indagación de las representaciones sociales sobre la paz y la guerra en sujetos que nacieron en una tierra con una historia de violencia y que al mismo tiempo hoy encuentran un país polarizado en razón del proceso de paz pero con expectativas de un futuro distinto. En consecuencia los niños, niñas y jóvenes (NNJ) se constituyen en los sujetos centrales de esta investigación.
A raíz o consecuencias de los hechos que incidieron en la violencia colombiana han ocasionado hasta la actualidad diversos conflictos sociales tales como el desplazamiento, muertes, inseguridad, deserción escolar, desempleo, vulneración de derechos humanos y calidad de vida, falta de dignidad humana, injusticia social, entre otros que afectan tanto directa como indirectamente a los NNJ.

Colombia se encuentra en un largo proceso de construcción de paz territorial y es por esta razón que abordar las Representaciones Sociales (RS) de guerra y paz son reto para proponer apuestas para 
la construcción de una cultura de" paces" a partir de la educación y la pedagogía.

Por otro lado, el desarrollo de esta investigación desde el macroproyecto de investigación en la línea de pedagogía para la paz y la convivencia de la Maestría en Práctica Pedagógica. Identifica como referente de estudio a los estudiantes del último curso de la institución educativa San José de Cúcuta capital de Norte de Santander, con el objetivo central de Comprender las RS de paz de los jóvenes como fundamento a una práctica pedagógica para la paz.

Este propósito integró elementos que posibilitaron el reconocimiento a la necesidad herramientas o estrategias que generan transformación de los conflictos presentes en aula de clases y promociono la convivencia en dicho escenario.

Además se permitió conocer las perspectivas de las condiciones sociales, políticas, culturales y económicas de Colombia en centros educativos y no solo como parte de la coyuntura mediática originada por la firma de los Acuerdos de Paz, sino también porque a través de ese reconocimiento se identifica que "en algunas sociedades no se ha dado el concepto de paz, principalmente porque se estaba de manera constante en guerra o preparándose para ella" (Jiménez, 2009, p.145).

Este postulado refleja lo que ha sido la evolución histórica del país y de la región, altamente influida por la tradición conservadora que orientó el desarrollo de una idiosincrasia frente a la forma de asumir y dar tratamiento al conflicto, la cual no da espacio a la inclusión, la tolerancia y la valoración de las diferencias que demanda la sociedad actual.

En ese sentido, este artículo presenta las principales reflexiones resultantes en la investigación previa que al nivel teórico se desarrollaron algunas categorías principales como los avances en los modelos pedagógicos desde el tradicional hasta el social y el holístico, la teoría de las Representaciones Sociales desarrollada por Moscovisci (1961) y Araya (2002) y una mirada de la hermenéutica en la práctica pedagógica Aristía, (2017).
Finalmente, se expone el resultado del estudio y sus aportes en la fundamentación de una práctica pedagógica social- critica.

\section{Metodología}

Con el fin de identificar las representaciones sociales de paz construidas por los niños y jóvenes, se hizo necesario abordar esta investigación desde un enfoque cualitativo- hermenéutico, con el cual se pretende interpretar los fenómenos tal como lo significaron los participantes.

En ese sentido para Hernández, Fernández y Baptista (2010) este enfoque permite "comprender y profundizar los fenómenos, explorándolos desde la perspectiva de los participantes en un ambiente natural y en relación con el contexto" (p. 12).

La investigación abarcó la relación que existe entre los significados de paz asignados por lo menores respecto la realidad del contexto educativo en el que se encuentran, el cual se caracteriza por ser un lugar marcado por la violencia.

Se aplicó un enfoque de carácter flexible, emergente e inductivo para el desarrollo del proceso, en esta dirección en la metodología condujo a interesarse por la realidad en el contexto directo en la que ocurre, razón por la cual las percepciones de quienes allí habitan son de gran interés y validez, de esta manera se comprendió e interpretó la problemática de estudio a partir de los datos recogidos y analizados desde una perspectiva holística y flexible que favorece interacciones más cercanas y genuinas.

Los criterios de selección se dieron dentro del marco del muestreo por conveniencia que de acuerdo con Hernandez et. al., (2010) "En ciertos estudios es necesaria la opinión de individuos expertos en un tema. Estas muestras son frecuentes en estudios cualitativos y exploratorios para generar hipótesis más precisas o la materia prima del diseño de cuestionarios" (p. 42).

Teniendo en cuenta lo anterior los participantes de interés, que pueden aportar convenientemente información $\mathrm{n}$ sobre el eje o tema central son 
estudiantes del colegio San José de Cúcuta, de lo cual se destaca que la muestra son estudiantes del grado once de dicha institución, es decir, una total de 11 estudiantes.

Las técnicas aplicadas para la recolección de la información fueron la entrevista semiestructurada, la observación no participante y el grupo focal.

Por su parte Janesick (1998) expone que en la entrevista, "a través de las preguntas y respuestas, se logra una comunicación y la construcción conjunta de significados respecto a un tema, esa es la finalidad que persigue la presente investigación al buscar conocer, relacionar y comprender las percepciones de los menores y jóvenes en torno a sus representaciones sociales".

Esta forma de observación es descrita por Hernandez et. al., (2010)" como la búsqueda de interpretación en la realidad, sin embargo en su variante de no participante el investigador asume una actitud de presencia, pero sin interferir en las acciones o decisiones que toman los participantes de la investigación".

"El grupo focal es una forma de interacción colectiva entre el investigador y un número determinado de sujetos". Según Duarte (2008):

Los grupos focales se realizaron con algunos de los jóvenes de Norte de Santander de la Institución Educativa San José de Cúcuta a fin de profundizar en elementos particulares encontrados en el análisis de la primera fase de codificación.

En ese sentido, para el análisis de la información cualitativa, se empleó la Teoría Fundamentada de Strauss y Corbin (2005) que define la codificación de la información en tres momentos:

Codificación abierta: se tomaron los resultados de la entrevista, la observación no participante y el grupo focal, como datos en formato de texto que fueron leídos y comparados línea por línea, para llegar a la identificación de hechos relevantes que facilitaron la agrupación de los datos. Codificación axial: en segundo lugar se establecieron categorías y subcategorías a través de una matriz de lectura, en la cual es posible precisar las categorías emergentes sobre las representaciones sociales. Codificación selectiva: finalmente, se seleccionan las grandes categorías en macro-categorías, de modo que facilitan una lectura hermenéutica de los hallazgos (p.7-8).

\section{Resultados}

Para la obtención de los resultados se tomó en cuenta como bases los modelos pedagógicos y la teoría de las representaciones sociales; teniendo en cuenta que esta investigación es cualitativa las herramientas de apoyo aplicadas fueron la entrevista semiestructurada y el grupo focal dirigida a los estudiantes de grado once la institución educativa San José de Cúcuta.

Para el análisis de los objetivos y generar aportes a estos se tuvo en cuenta la perspectiva de la práctica pedagógica ya que permite conocer y ampliar el estudio o la interpretación mediante la observación del individuo desde diferentes enfoques, en lo social, cultural, cognitivo, afectivo, entre otros.

Teniendo este orden de ideas, se presentarán a continuación los resultados obtenidos en esta investigación con su respectiva interpretación de acuerdo con los objetivos planteados.

Para los estudiantes quienes en su mayoría mencionaban que la paz es el reflejo de la tranquilidad en sus hogares. Es decir, mencionaban que la paz es un estado de tranquilidad que inicia desde la funcionalidad y ambiente familiar pese a la circunstancias y conflictos o crisis que se puedan manifestar en el microsistema, asimismo, manifiestan que el rendimiento académico hace parte de ese bienestar o "ese sentirse bien" que ellos definen como paz.

Además, recalcan que la paz es la ausencia de guerras, de conflictos y de la violencia, el objeto de estudios es reconocido como la alteración en la convivencia familiar y comunitaria (vecinos) e incluyen el desequilibrio económico dentro de la familiar o por otro lado, es la actitud que se tenga frente a una situación de desacuerdo en cualquier ambiente, ya sea el laboral, el escolar o familiar; desde otro ámbito. 
Para los estudiantes la paz es sinónimo de respeto, de unión, de libertad, de responsabilidad, amor, comprensión y cada uno de los valores que puedan existir, la paz es propia de valores, debe fundamentarla en los mismos y ser un agente de suma importación para la sociedad.

Asimismo, paz es relacionada como los acuerdos de paz en la nación colombiana, la corrupción, los grupos al margen de la ley que generan daño social con los secuestros, muertes, despojo de tierras e incumplimiento a los acuerdos; igualmente declaran que la paz ha sido vulnerada durante los gobiernos de los expresidentes Santos y Uribe, pues ha ocasionado confusión al pueblo colombiano, por ejemplo: los falsos positivos, la inseguridad o falta de coherencia del planteamiento del acuerdo de paz.

Para los estudiantes no es posible lograr una paz en Colombia por la existencia de grupos armados al margen de la ley. Por otro lado los medios de comunicación colombianos en este caso los canales televisivos como RCN y CARACOL, como también redes sociales como Facebook, son la principal fuente de información suministrada a los jóvenes entorno a la paz colombiana. Aunque la institución educativa orienta a la investigación de lo relacionado con la historia conflictiva de Colombia o la paz.

Durante la aplicación del grupo focal se conoció que los jóvenes definen tres tipos de paz, la espiritual o personal, familiar y la social, en la cual la primera hace énfasis al estado armónico con sí mismo, el tener la conciencia limpia; la paz familiar se relaciona con la superación de crisis mediante el dialogo, la interacción y lazos afectivos entre padre, hijo y hermanos; mientras que la paz social es todo lo relacionado con la estabilidad o el bienestar en la sociedad y la comunidad.

De esta manera se identificó que los estudiantes participantes de la investigación expresan que la comunicación, el dialogo y la educación son los principales agentes o factores de intervención en gestión de la paz, no solo en el ámbito familiar sino también en la cotidianidad implicando diferentes escenarios, el barrio, el colegio, el trabajo y de esta manera llegar a mejorar la sociedad.
Seguidamente se analizó las Representaciones Sociales en los Jóvenes estudiantes de la institución educativa San José de Cúcuta, en este orden de ideas se analiza cada una de las entrevistas realizadas a los estudiantes de grado once de la institución educativa San José de Cúcuta.

En primer lugar los estudiantes tienen una idea o definición general de la paz relacionada como la relación con sus padres, sin embargo es importante resaltar que la tipología familiar más relevante es la monoparental, es decir viven con uno de sus padres, en este caso la madre; como se mencionó con anterioridad, lo jóvenes relacionan que el primer acercamiento o el lugar en el que debe haber mayor estado de tranquilidad, armonía y convivencia en sus casas, con sus familias.

En otras palabras, la funcionalidad familiar, la cohesión o lazos familiares, los roles propios en la familia, las reglas, la confianza, la comunicación, la adopción de valores o el buen clima- ambiente familiar, permite un primer acercamiento a la paz, de ahí el aprender a desarrollarla y aplicarla en los demás escenarios de interacción

Los jóvenes objeto de estudio entienden la paz cómo un agente de equilibrio y bienestar, es un factor que pueden resolver de forma pacífica las diferencias y que genera una dignidad humana o es un ingrediente esencial para vivenciar la misma. Dicho esto, se conoció que los jóvenes no expusieron ningún hecho de paz dentro de los establecimientos escolares, más bien, lo relacionaron con la situación social colombiana, su historia violenta y conflictiva que hace parte la política.

Por tanto, las representaciones de la paz en los jóvenes se han estructurado o jerarquizado mediante la experiencia familiar y la influencia de la cultura, la sociedad y los medios de comunicación, esto implica la interpretación de eventos desafortunados como el conflicto armado en Colombia.

Entre tanto, para ellos es más sencillo apropiarse y de lograr la paz en sus familias que en lo social o el país, para ellos socialmente la paz es incansable lo que ha permitido una actitud sin esperanza ante los acuerdos de paz y negativa o de rechazo ante gobierno nacional. 
El estudio de las representaciones sociales desarrolló enfoques teóricos que complementan la visión de Moscovici, el modelo socio-genético denominado también como enfoque clásico por este autor es el punto de partida, pero también se consideran los aportes del modelo estructural que aborda la teoría del núcleo central con autores como Flament o Guimelli y el modelo socio-dinámico, denominado la Escuela de Ginebra, liderada por el investigador Doise.

Partiendo de lo anterior, se establece que los enfoques están ligados, por tanto, el pensamiento social cambia la realidad, vemos como en perspectiva de la paz desde la conclusión social se relaciona con los acuerdos de paz y la relación del conflicto armado con el gobierno y el país. Es así como se captan y estructuran conocimientos que dan una representación en los jóvenes y en las nuevas generaciones, pues dicha definición es captada desde siglos atrás en la historia colombiana y por un sistema de valores y normas sociales que por consiguiente se da una relación con el sujeto o el grupo con el fenómeno (paz).

En el enfoque desarrollado por Doise, expone que no existe una representación única que sea común para los individuos de un grupo. Sin embargo, existe una meta o un sistema normativo que estructura el pensamiento representativo del individuo; que está constituido por saberes que pertenecen a los grupos dominantes de cualquier contexto social y cultural.

Es decir que as representaciones sociales son adaptadas y se pueden moldear, es así como se observa que los estudiantes definen la paz desde la influencia de medios de relación o medios de comunicación, los cuales estructuran los pensamientos desde la información que dan al sistema social y se logra a pesar de las diferentes interpretaciones una "meta" común de funciones o una representación compartida.

Generar aportes que fundamenten una práctica pedagógica para la paz

Inicialmente se sustenta que se apoyó de los modelos pedagógicos y de la base teórica una mirada desde la hermenéutica de la práctica pedagógica, además se implementó un recurso didáctico que permite dar cumpliendo a este objetivo.

Tomando en cuenta los fundamentos teóricos se interpreta la práctica pedagógica como un elemento esencial para la reconstrucción del tejido social, pues no solo va en pro del área cognitivo de los individuos sino que también abarca lo social, afectivo y cultural, es decir, no deja de lado la esencia total de la persona.

Esta disciplina tiene un enfoque de desarrollo social por medio de la educación, durante décadas ha relacionado al alumno - maestro, mediante proceso de enseñanza-aprendizaje, es por eso que esta práctica es un excelente factor para aplicar, enseñar, orientar, instruir y hasta reestructurar la paz o el objeto de este estudio.

Uno de las labores más sobresalientes de un maestro es encarrilar y disciplinar al estudiante con aportes significativos para su aprendizaje, por tanto es indiscutiblemente apropiado como instrumento que genere cambios cognitivos y afectivos en diferentes temas que enriquezca al estudiante, en este caso la paz y sus representaciones sociales.

Según reflexiona De Zubiria (2007) "No se trata simplemente de transmitir conocimientos, como supuso equivocadamente la Escuela Tradicional, sino de formar individuos más inteligentes a nivel cognitivo, afectivo y práxico. No se trata-por bonitas que suenen las palabras-de hacer sentir feliz al niño y al joven; se trata de formarlo con toda la felicidad, esfuerzo, cuidado, responsabilidad, diálogo y trabajo que ello demanda" (p.8).

Es por ello que desde la practica pedagógica, permite que el individuo tome conciencia y sea autónomo de sus decisiones, sin embargo, este paradigma da un soporte para que el estudiante sea determinante y tome iniciativa en los aspectos más necesarios para la reconstrucción o desarrollo social.

En este orden de ideas se implementó la catilla "la paz en el barrio" (anexo1), donde están contenidos aspectos a desarrollar tales como la coyuntura del país, jóvenes en la construcción de paz, NNAJ sujetos dentro de la práctica pedagógica y Práctica pedagógica desde una mirada hermenéutica. 
Comprender las Representaciones Sociales de paz de los jóvenes estudiantes de la institución educativa San José de Cúcuta como fundamento a una práctica pedagógica

Se tomó como base de respuesta a este objetivo una mirada hermenéutica a la práctica pedagógica que requiere iniciar un abordaje desde la Teoría de las Prácticas Sociales (TPS), misma que agrupa "aquellas teorías que las definen como el componente fundamental del mundo social" (Aristía, 2017, p.221). TPS “antepone la práctica como principal unidad de compresión y generación del mundo social" (Aristía, 2017, p.223

De tal manera que para entender las prácticas sociales hay que tener en cuenta tres dimensiones, la primera es la sociológica que sustenta que es la relación entre la estructura social, los actores sociales es mediante los hábitos cotidianos. La segunda etnometodológica que presenta las situaciones empíricas como aquellas que dan lugar a lo social, comprende el mundo social como un resultado de la práctica soportado en conocimiento que se produce y circula en el sistema. Y por último la comprensión de lo social desde la praxis ya que el mundo social es resultado práctico de formas de actividad.

Por tanto es argumentativo decir que la práctica permitirá entender las representaciones sociales de la paz en los jóvenes estudiantes de este estudio, pues son las acciones individuales y la capacidad de las instituciones de incidir en el mundo, en este punto es necesario retomar que los actores de estudio, reconocer algunos aspectos de la paz desde los medios de comunicación, siendo estos una gran masa global de influencia en la estructura social.

Ahora bien, es indispensable lograr el cumplimento de este objetivo desde la pedagogía o como pedagogos, percibiendo la reflexividad como agente fundamental para la comprensión de este aspecto de investigación, para ello se debe tener en cuenta que las representaciones sociales son producidas por una clasificación especifica según el autor Bourdieu y Wacquant, los cuales son los esquemas mentales y corporales, las conductas, los pensamientos, los sentimientos, y juicios de los agentes o actores sociales.

Además de tres elementos, competencias, sentido y materialidades; las competencias son los saberes, el sentido como las emociones y valor al objeto de manera cultural y la materialidad que abarca la totalidad de las herramientas, infraestructuras y recursos que participan que aportan información,

Concluyendo lo anterior, para comprender las representaciones sociales de la paz en los jóvenes de la institución educativa San José de Cúcuta, hay que identificarlos como agentes sociales independientes con distintas capacidades de interpretación de la realidad debido a su individualidad de sentidos, capacidades, mentalidades, emociones y cultura;

Sin embargo, llegan a un mismo nivel o meta en la definición de la paz pues esta se incluye como práctica cotidiana incluida por estructura social y a pesar de seres individuales en la codificación y recepción del sistema social los medios masivos de influencia logran dar un conjunto de saberes interconectados.

\section{Discusiones}

Los estudios demostraron que la paz constituye una de los factores más esenciales para desarrollo social, Colombia es una nación con décadas de guerra, conflictividad, violencia social y corrupción que ha conllevando a quebrantamientos de los derechos humanos la calidad de vida, la dignidad humana y por tanto ha vulneradora justicia social y la paz entendida más que como la ausencia de conflicto, la capacidad y la actitud de superarlos con la paz, la tolerancia, el respeto, trayendo consigo la equidad, igualdad, libertad, justicia y tranquilidad.

Según los reportes de los resultados obtenidos y al dar respuesta preguntan de investigación, las representaciones sociales de la paz en los jóvenes está vinculada primeramente al sano ambiente en dinámica y la funcionalidad no solo en familiar sino también los pequeños escenarios de interacción como el colegio, el barrio y posteriormente la sociedad.

Por otro lado, los aportes de la educación y de la pedagogía ayudan a crear directrices prácticas para 
crear un vínculo de enseñanza cognitiva, y además el crecimiento y enriquecimiento de los estudiantes, dando conocimientos críticos y fundamentales en su total progreso personal, cultural y social para que sean agentes promotores de paz

\section{Conclusiones}

A partir de los datos obtenidos se concluyen los siguientes criterios:

Los conocimientos de las representaciones sociales en los jóvenes estudiantes están siendo influenciadas por medios de comunicación que a su vez no son completamente sustentables para poder determinar una realidad social, es decir, que los conocimientos y cimientos de la sociedad también debe estar estructurados mediante paradigmas, teorías, disciplinas y/o saberes cognitivos que de una amplitud de los escenarios sociales y la definición de estos.

La paz es reconocida como factor esencial para las interacciones de los individuos con el medio y se comprende que el mismo medio interviene e influye en el entendimiento, interpretación de las realidades sociales, formando una representación común en los individuos, de aquí se comprende los factores, las conclusiones y saberes epistemológicos que inciden en ella .

Finalmente se reconoce la pedagogía como agente reconstructor del tejido social y fomentador de individuos capaces de superar el conflicto, la violencia, las crisis, las desigualdades y de forjar nuevos contextos formados con integridad, vínculos saludable, con mayor bienestar y justicia social.

\section{Referencias}

Abric, J. C. (1994). Prácticas sociales y representaciones. México: Ediciones Coyoacán,

Araya Umaña, S. (2002). Las representaciones sociales: Ejes teóricos para su discusióncuaderno de ciencias sociales 127. Costa Rica: Flacso (Facultad Latinoamericana De Ciencias Sociales)
Bautista, F. J. (2014). Paz neutra: Una ilustración del concepto. Revista De Paz y Conflictos, (7), 1952.

Caballero, A. (2002). Educación para la paz y la convivencia: Implicaciones para la gestión de conflictos escolares. Cultura y Educación, 14(4), 403-411.

Cerdas-Agüero, E. (2015). Desafíos de la educación para la paz hacia la construcción de una cultura de paz. Revista Electrónica Educare, 19(2), 135-154.

Cerdas-Agüero, E. (2015). Desafíos de la educación para la paz hacia la construcción de una cultura de paz. Revista Electrónica Educare, 19(2), 135-154.

De la Cruz Vergara, Maribel, Guillén, A., Guillén, A., \& Weber, J. R. Apreciado Eduardo, he leído esta versión y no tengo sugerencias, de otra parte me gustaría presentar la propuesta a mi universidad, para ello es necesario que me informes sobre las implicaciones de alojar el proyecto, sobre todo económicas, ya que es la primera pregunta que me haría el rector.

De Vera, F. H. (2016). La construcción del concepto de paz: Paz negativa, paz positiva y paz imperfecta. Cuadernos De Estrategia, (183), 119-146.

De Vera, F. H. (2016). La construcción del concepto de paz: Paz negativa, paz positiva y paz imperfecta. Cuadernos De Estrategia, (183), 119-146.

De Zubiría, J. (2007). Modelos pedagógicos contemporáneos. Editorial Magisterio.Bogotá,

Díaz, A. M., \& Torres, F. J. S. (2004). Geografía de los cultivos ilícitos y conflicto armado en Colombia Universidad de los Andes, Facultad de Economía, CEDE.

Fisas, V. (2011). Educar para una cultura de paz. Quaderns De Construcción De Pau, 20, 7-24.

Gómez, L. E. C., \& Alvarado, F. G. (2008). Repensar la educación y la pedagogía: Algunas reflexiones críticas. Revista Electrónica Educare, 12(2), 4764.

Hernández Carrera, R. M. (2014). La investigación cualitativa a través de entrevistas: Su análisis 
mediante la teoría fundamentada. Cuestiones Pedagógicas, 23, 187-210,

Jiménez Bautista, F. (2009). Hacia un paradigma pacífico: La paz neutra.

López Gartner, R. N. (2016). Representaciones De Violencia y Paz Que Los Niños y Las Niñas Significan a Través De Los Noticieros De Televisión: Estudio Con Alumnos y Alumnas De Grado $5^{\circ}$ De Primaria Del Instituto Cultural Riosucio,

Magaloni, B., Calderón, G., Diaz-Cayeros, A., Robles, G., \& Olarte, J. (2013). The beheading of criminal organizations and the dynamics of violence in Mexico's drug war. Unpublished Manuscript, Stanford University,

Márquez, A. I. (2014). El papel de la educación en situaciones de posconflicto: Estrategias y recomendaciones. Hallazgos, 11(21)

Materán, A. (2008). Las representaciones sociales: Un referente teórico para la investigación educativa. Geoenseñanza, 13(2), 243-248.

Mireles Vargas, O. (2011). Representaciones sociales: Debates y atributos para el estudio de la educación. Sinéctica, (36), 1-11.

Muñoz, F. (2001). La paz imperfecta ante un universo en conflicto. La Paz Imperfecta, , 21-66.

PNUD, C., \& en México, G. (2003). Programa de las naciones unidas para el desarrollo.

Ramos, C., Nieto, A. M., \& Chaux, E. (2007). Aulas en paz. Revista Interamericana De Educación Para La Democracia, 1(1), 36-56.

Villalobos, Y. S. (2018). Los instrumentos de la catedra de paz, como estrategia en la reconstrucción social de una nueva Colombia. Revista Experiencia Docente, 5(1), 19-35. 